\title{
Current Guidelines in the Management of Upper Gastrointestinal Subepithelial Tumors
}

\author{
Jin Woong Cho and the Korean ESD Study Group \\ Department of Internal Medicine, Presbyterian Medical Center, Jeonju, Korea
}

Subepithelial tumors are frequently found in asymptomatic patients in Japan and Korea where cancer screening tests routinely include endoscopy. Most lesions are asymptomatic and clinically insignificant. However, carcinoid tumors, lymphomas, glomus tumor and gastrointestinal stromal tumors (GISTs) are malignant or have the potential to become malignant. Inflammation due to parasitic infestation by Anisakis and poorly differentiated adenocarcinomas in the stomach rarely present as subepithelial lesions. In contrast to the frequency of gastric GIST in the gastrointestinal system, they are uncommon in the duodenum and very rare in the esophagus. The prognosis of patients with GISTs in the stomach is relatively good compared with GISTs in other organs. Along with the location of the tumor, its size and mitotic count are major factors that determine the malignant potential of GIST. Small $(<2 \mathrm{~cm})$ asymptomatic GISTs usually have benign clinical course. GIST is the most common subepithelial tumor to occur in the stomach. Although various methods are employed to diagnose GISTs, the risk of GIST metastasis cannot be accurately predicted before lesions are completely resected. Recently, new endoscopic diagnostic methods and treatment techniques have been developed that allow the diagnosis and resection of lesions located in the muscularis propria, without any complications. These endoscopic methods have different indications depending on regions where they are performed. Clin Endosc 2016;49:235-240

Key Words: Stomach neoplasms; Gastrointestinal stromal tumors; Endosonography

\section{INTRODUCTION}

Subepithelial tumors (SETs) that occur in the gastrointestinal tract include all lesions with smooth prominentia in the inner cavity of the gastrointestinal tract and no change in the mucosal surface on endoscopy. SET were previously called submucosal tumors and they are frequently detected in patients during cancer screening examinations. The term "SET" includes lesions in the mucosal layer that grow under the epithelium. SETs are classified into non-neoplastic and neoplastic lesions. Most lesions are asymptomatic and clinically insignificant. However, carcinoid tumors, lymphomas, glomus

Received: July 25, 2015 Revised: October 10, 2015

Accepted: December 22, 2015

Correspondence: Jin Woong Cho

Department of Internal Medicine, Presbyterian Medical Center, 365 Seowon-ro, Wansan-gu, Jeonju 54987, Korea

Tel: +82-63-230-1321, Fax: +82-63-230-1309, E-mail: jeja-1004@hanmail.net

(c) This is an Open Access article distributed under the terms of the Creative Commons Attribution Non-Commercial License (http://creativecommons.org/ licenses/by-nc/3.0) which permits unrestricted non-commercial use, distribution, and reproduction in any medium, provided the original work is properly cited. tumors, and gastrointestinal stromal tumors (GISTs) are malignant or have the potential to become malignant. Even with advanced endoscopic facilities and abundant research, SETs are still very difficult to diagnose with noninvasive methods, such as endoscopy or endoscopic ultrasonography (EUS), before surgery. There are various methods available for histological diagnosis of the tumor, including bite-on-bite biopsy, EUS-guided fine-needle aspiration (EUS-FNA), endoscopic mucosal resection (EMR), endoscopic submucosal dissection (ESD), and submucosal tunneling with endoscopic resection (STER). To find and treat malignant lesions at an early stage, appropriate diagnostic approaches are necessary, depending on the characteristics of the lesions.

\section{CLINICAL CHARACTERISTICS AND FOLLOW-UP RESULTS OF SUBEPITHELIAL LESIONS}

Subepithelial lesions are classified into non-neoplastic le- 

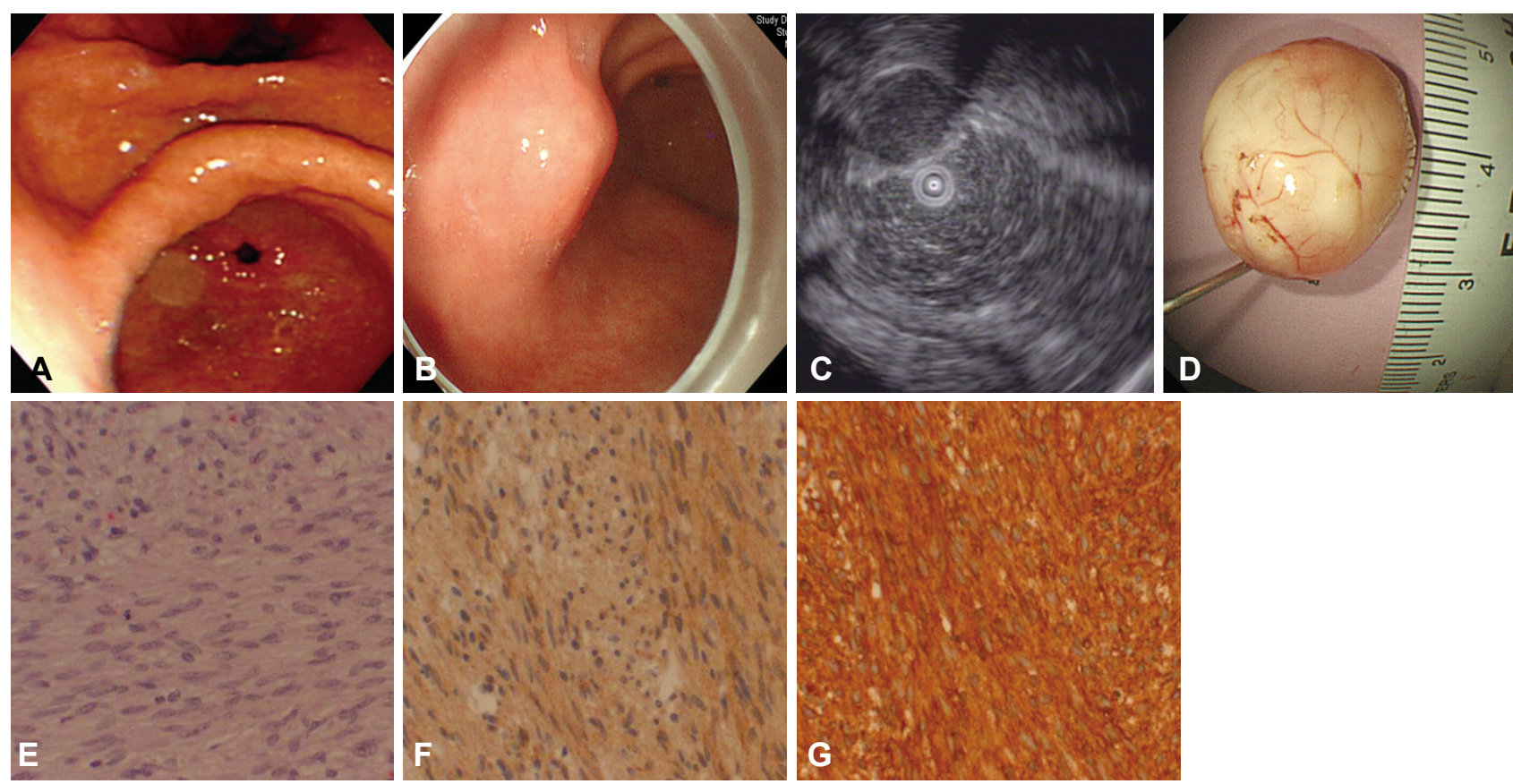

Fig. 1. Gastric gastrointestinal stromal tumor (GIST) with rapid growing. (A) Endoscopy didn't detect any mass in the stomach. (B) Four years later, endoscopy showed round smooth elevated mass in gastric angle. (C, D) Enodoscopic subtumorial resection was performed. Tumor size was $1.7 \mathrm{~cm}$. Microscopic finding. GIST with spindle cell type had 7 mitoses/50 high powered fields. $\mathrm{H} \& \mathrm{E}$ stain $(\mathrm{E}, \times 200)$ was done, and immunohistochemical stains were for positive for $\mathrm{c}-\mathrm{kit}(\mathrm{F}, \times 200)$ and $\mathrm{CD} 34(\mathrm{G}, \times 200)$.

sions, including inflammation, cysts, and an ectopic pancreas; and neoplastic lesions, such as GISTs, carcinoid tumors, leiomyomas, lymphomas, schwannomas, glomus tumors, and lymphangiomas. Inflammation due to parasitic infestation by Anisakis and poorly differentiated adenocarcinomas in the stomach rarely present as subepithelial lesions.

SETs are frequently found in asymptomatic patients in Japan and Korea where cancer screening tests routinely include endoscopy. In follow-up studies of asymptomatic upper gastrointestinal tract subepithelial lesions, the lesions increased in size in fewer than $10 \%$ of patients. Among patients who had health screening tests, Lim et al. ${ }^{1}$ reported that $795(0.76 \%)$ had subepithelial lesions. They conducted a follow-up study of 252 patients for an average of 84 months using endoscopy. The size of the lesions increased in only eight (3.2\%) of the patients. Gill et al. ${ }^{2}$ reported a study of 51 asymptomatic patients with subepithelial lesions $<3 \mathrm{~cm}$ in the upper gastrointestinal tract who were followed for 29.7 months, on average, using EUS. The lesions changed in size or echogenicity in only seven (13.7\%) of the patients. Among tumors that originated from the fourth layer, the lesions increased in size in five patients $(12 \%){ }^{2}$ Kim et al. ${ }^{3}$ evaluated 948 patients in Korea with 989 gastric SETs $<30 \mathrm{~mm}$ in size using endoscopy or EUS. The changes of lesions were significant in size, echogenicity, or surface integrity in $84(8.5 \%)$ of the patients, and 21 showed alterations in echo patterns and size. Among 25 patients who underwent resection through surgery or endoscopy, 19 patients were diagnosed with a GIST (Fig. 1). ${ }^{3}$

\section{GASTROINTESTINAL STROMAL TUMORS}

\section{Clinical manifestation and prognosis}

Most SETs can be classified as malignant or benign at the time of the diagnosis. Although GISTs have the potential to become malignant, small asymptomatic lesions appear as benign tumors. GISTs are the most common type of SET in the stomach. Kawanowa et al. ${ }^{4}$ analyzed resected stomachs and found 50 GISTs in 35 patients, with $90 \%$ of the tumors located in the upper stomach. Considering the frequency of GISTs observed in the clinic, only a small number of microscopic GISTs seem to show changes, such as an increase in size. ${ }^{4}$ Agaimy et al. ${ }^{5}$ reported that microscopic GISTs are not common in intestinal resections. Therefore, the detection frequency of GISTs appears to depend on their location in the gastrointestinal tract. ${ }^{5}$

In contrast to the high frequency of GISTs in the stomach, they are uncommon in the duodenum and very rare in the esophagus. The prognosis of patients with GISTs in the stomach is relatively good compared with GISTs in other organs. ${ }^{6,7}$ Along with the location of the tumor, its size and mitotic 
count are major factors that determine the malignant potential of GISTs. ${ }^{6}$ Miettinen et al. ${ }^{6}$ reported the characteristics of 1,765 cases of stomach GISTs. Only 2.7\% developed before the age of 21 , and the most common symptom was gastrointestinal bleeding. In their study, the size of the tumors and their mitotic counts were important factors in the long-term prognosis. When a tumor was $<10 \mathrm{~cm}$ or the mitotic count was $<5 / 50$ high powered fields (HPFs), only $2 \%$ to $3 \%$ had metastasized. However, in tumors $>10 \mathrm{~cm}$ and in those with a mitotic count $>5 / 50 \mathrm{HPFs}, 86 \%$ had metastasized. ${ }^{8}$ All GISTs that occurred in the intestines had more than a moderate possibility of metastasis when they were $>5 \mathrm{~cm}$ or had $>5$ mitoses $/ 50$ HPFs. In tumors $<5 \mathrm{~cm}$ with a mitotic count $<5 / 50 \mathrm{HPFs}$, the intestinal GISTs had a low probability of metastasis. ${ }^{6}$

Other than the size of the tumor and the mitotic count, mucosal disruption, necrosis, high cellularity, and tumor rupture are additional pathological factors that affect GIST malignancy. ${ }^{9,10}$ Trupiano et al. ${ }^{11}$ divided 77 GIST patients into an adverse outcome group and a non-adverse outcome group. They suggested that the following were associated with malignancy: a tumor larger than $7 \mathrm{~cm}$, high cellularity, mucosal invasion, a high nuclear grade, more than 5 mitoses/50 HPFs, a mixed cell type, the existence of a myxoid background, and the absence of stromal hyalinization. Using these factors, for diagnosing malignancy the sensitivity was $100 \%$ and the specificity was $92 \%$, showing a high predictive value.

Nilsson et al. ${ }^{12}$ analyzed 288 patients with GIST based on a risk classification system proposed in 2002. There were only two (1.2\%) tumor-related deaths among the patients classified as having a very low risk, a low risk, or an intermediate risk. In contrast, among those classified as having high-risk and malignant tumors, $63 \%$ and $83 \%$, respectively, experienced tumor-related deaths. ${ }^{12}$

\section{Treatment approach}

A National Comprehensive Cancer Network (NCCN) guideline in 2010 suggested different treatments for gastrointestinal tract GISTs with malignant potential, depending on their location, size, and mitotic counts. According to the NCCN, if the GIST was $>2$ or $<2 \mathrm{~cm}$ with symptoms, it should be removed. It noted that patients with incidentally discovered GISTs $<2 \mathrm{~cm}$ should first undergo EUS-FNA or an abdominopelvic computed tomography with contrast enhancement (CECT). If the EUS revealed a high-risk tumor (irregular border, cystic space, ulceration, echogenic foci, and heterogeneity), it should be surgically removed. If there were no signs indicating high risk, the GIST should be followed up at 6 to 12 months intervals. ${ }^{13,14}$ In 2014, the European Society for Medical Oncology (ESMO) group proposed that histologically diagnosed small GISTs should be removed. ${ }^{15}$ They based this proposal on previous studies showing that tumors $<2 \mathrm{~cm}$ with $<5$ mitoses/50 HPFs can metastasize in very rare cases, even though they are classified as very low risk..$^{10,16}$

\section{Endoscopy and EUS}

According to an earlier study, gastric GISTs located in the gastroesophageal junction or fundus or those that exhibit coagulation necrosis, ulceration, or mucosal invasion have a poor prognosis, whereas those located in the antrum have a good prognosis. ${ }^{8}$ Another study reported that GISTs with irregular borders or tumorous ulcers on endoscopy have the potential to become malignant. ${ }^{17}$

According to the EUS guideline of the American Society of Gastrointestinal Endoscopy (ASGE) in 2007, EUS is the most valuable test for evaluating the size, margin, layer of origin, and echogenicity of lesions. ${ }^{18}$ However, the accuracy of EUS in diagnosing subepithelial lesions is relatively low (46\% to $48 \%$ ). According to Hwang et al., ${ }^{19}$ masses present in the third and fourth layers are more difficult to diagnose..$^{20}$ EUS is the most accurate test to distinguish the layer where a lesion is located. Its internal echo pattern is also very useful for deciding the course of treatment. ${ }^{14}$

High-risk lesions on EUS in the 2013 Japan Gastroenterological Endoscopy Society (JGES) guidelines are those with irregular borders and internal heterogeneity, including anechoic areas (necrosis) and echogenic foci (bleeding), heterogeneous enhancement, and regional lymph node enlargement. ${ }^{17,21}$ ASGE did not agree on the value of some features, such as echogenic foci, anechoic space, and heterogeneous echotexture. $^{18}$

\section{DIAGNOSTIC APPROACH TO TUMORS LOCATED IN THE MUCOSAL AND SUBMUCOSAL LAYER}

Most lesions classified as subepithelial are located in the mucosal and submucosal layers and are asymptomatic. However, malignant adenocarcinomas, lymphomas, or tumors with metastasis can result in symptoms. Patients with Anisakis infestation in the stomach present with upper abdominal pain in both the acute and chronic phases.

Subepithelial lesions can be diagnosed by endoscope and EUS, although the accuracy of the diagnosis depends on the skill of the endoscopist. In some lesions, EMR or EUS-FNA may be helpful in the diagnosis. In 2011, the guideline of the European Society of Gastrointestinal Endoscopy (ESGE) suggested that a bite-on-bite biopsy should first be conducted, followed by an endoscopic resection. Patients with suspected lymphomas, neuroendocrine tumors, or extrinsic tumors on 
EUS should be managed with EUS-guided FNA or a biopsy. ${ }^{22}$ ASGE recommended that the decision to perform EUS surveillance with asymptomatic small submucosal tumors should be individualized because EUS surveillance has not been validated. ${ }^{18}$ Khashab and Pasricha ${ }^{15}$ proposed, in an editorial in Gastrointestinal Endoscopy in 2013, that symptomatic lesions or lesions that increase in size should be resected using EMR or ESD. For asymptomatic lesions or those that do not change in size, lipomas, vascular lesions, or cysts can be diagnosed only by using EUS, and other types of lesions should be evaluated histologically by one of the following: EUS-FNA, a biteon-bite biopsy, an EUS core biopsy, an unroofing technique, a single-incision needle-knife biopsy, submucosal endoscopy with a core biopsy, or a jumbo biopsy. If not enough tissues are acquired with these methods, EMR, ESD, or a gastrofiberscopic follow-up should be performed. ${ }^{15}$

\section{APPROACH TO TUMORS LOCATED IN THE MUSCULARIS PROPRIA LAYER}

GISTs, leiomyomas, and schwannomas are located in the fourth layer on EUS. Occasionally, an ectopic pancreas may invade the third and fourth layer. GISTs are most common in the stomach, and leiomyomas are frequent in the esophagus. In terms of the diagnostic approach, it is very important to distinguish a GIST from other types of tumors, to precisely judge the malignant potential of the tumor, and decide how to treat it. The accuracy of the diagnosis was reported to be less than $50 \%$ when using only EUS. Invasive tests, such as EUSFNA, have been reported to have a diagnostic yield of over $90 \%$. However, it is difficult to measure and interpret the level of mitoses in a GIST using FNA or various biopsy techniques because the cellularity and the mitotic count of GISTs are different according to the site within the tumor. The fixation time and the type of fixative can also affect measurements of mitoses. ${ }^{11}$

\section{Tumors less than $2 \mathrm{~cm}$}

Miettinen et al. ${ }^{6}$ reported the long-term prognosis of GISTs. Tumors $<2 \mathrm{~cm}$ did not metastasize if the number of mitoses was less than 5/50 HPFs. However, GISTs with a mitotic count greater than 6/50 HPFs showed a high level of metastasis in all gastrointestinal tract tumors, except the stomach. In 2013, JGES recommended the use of EUS, EUS-FNA, CECT, and surgery for gastric SETs $<2 \mathrm{~cm}$ suggestive of malignancy (an irregular border or a tumorous ulcer) on endoscopy. ${ }^{17}$ They recommend that lesions not considered malignant should be followed up every year or two using endoscopy or EUS. The ESMO and the ESGE suggest performing EUS 3 months after the detection of SETs $<2 \mathrm{~cm}$ in the esophagus, stomach, and duodenum, followed by a yearly follow-up thereafter. If the lesions increase in size or became symptomatic, they should be removed. ${ }^{22,23}$ In cases of asymptomatic subepithelial lesions $<2$ $\mathrm{cm}$ in the gastrointestinal tract that show no changes in size, Khashab and Pasricha ${ }^{15}$ suggested that a histological diagnosis should be attempted using various methods.

\section{Tumors larger than $2 \mathrm{~cm}$}

Although GISTs between 2 and $5 \mathrm{~cm}$ with mitoses less than 5/50 HPFs were reported to have a low probability (less than $10 \%$ ) of metastasis, the risk of metastasis increased to $16 \%$ to $73 \%$ when the mitotic count increased. ${ }^{6}$ Khashab and Pasricha $^{15}$ suggested STER or curative resection via surgery when the tumor is between 2 and $4 \mathrm{~cm}$, is symptomatic, or increases in size. According to the ESGE, laparoscopic wedge resection is the best treatment method. As the diagnostic accuracy and evaluation of mitoses are limited with EUS-FNA and biopsies, the ESGE recommends these only for GISTs with a high surgical risk, tumors located in the cardia or esophagus, or unresectable GISTs. ${ }^{18,22}$ The JGES also recommends surgical resection for gastric subepithelial lesions between 2 and $5 \mathrm{~cm} .^{17}$ In 2008, the Japan Society of Clinical Oncology suggested that tumors greater than $5 \mathrm{~cm}$ were an indication for surgery. ${ }^{24}$ They recommend EUS, EUS-FNA, or CECT for gastric lesions 2 to $5 \mathrm{~cm}$. In addition, they state that if a biopsy result indicates that the lesion is not a GIST, the doctor should choose a treatment method according to the type of disease. If endoscopy and CECT did not suggest malignancy (necrosis, hemorrhage, irregularity of the margin, abundant blood flow), they advised that the lesion could be operated upon using endoscopic surgery or periodically observed.

\section{SUGGESTED ALGORITHM IN THE ENDOSCOPIC APPROACH TO GASTRIC SUBEPITHELIAL TUMORS}

Asymptomatic subepithelial lesions smaller than $2 \mathrm{~cm}$ usually have a benign course, and it is recommended that they be managed by periodic surveillance using endoscopy or EUS. GIST proven by biopsy should be removed completely regardless of tumor size. Gastric subepithelial lesions with lesion-specific symptoms or those increasing in size may have malignant potential or active inflammation. Also, malignant features on endoscopy or high-risk features on EUS means a high probability of a clinically malignant condition. Biopsy or resection of the mass is needed for accurate determination of the long-term prognosis (Fig. 2). 


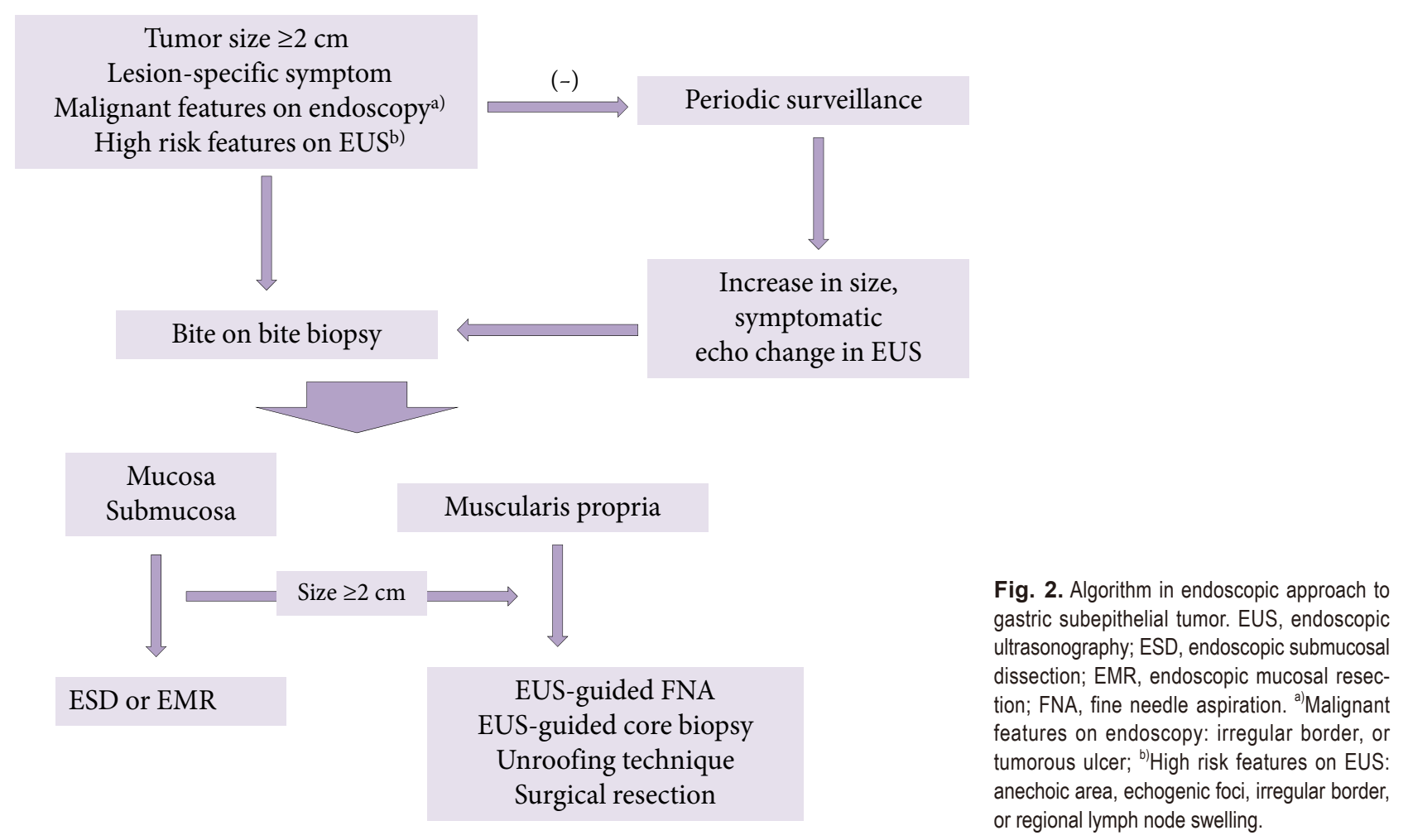

\section{CONCLUSIONS}

Subepithelial lesions include various neoplastic and non-neoplastic tumors. Most of these are benign. Endoscopy and EUS are helpful to distinguish malignant tumors. Although GISTs have the potential to become malignant, small $(<2 \mathrm{~cm})$ asymptomatic tumors usually have a benign clinical course. GIST is the most common SET to occur in the stomach. Although various methods are employed to diagnose GISTs, the risk of GIST metastasis cannot be accurately predicted before lesions are completely resected.

Recently, new endoscopic diagnostic methods and treatment techniques have been developed that allow for the diagnosis and resection of lesions located in the muscularis propria, without any complications. These endoscopic methods have different guidelines depending on the regions where they are performed because the experiences of endoscopic surgeons are different in resection of subepithelial lesions. The role of endoscopy has expanded to include not only the diagnosis of subepithelial lesions but also their treatment.

\section{Conflicts of Interest}

The authors have no financial conflicts of interest.

\section{REFERENCES}

1. Lim YJ, Son HJ, Lee JS, et al. Clinical course of subepithelial lesions detected on upper gastrointestinal endoscopy. World J Gastroenterol 2010;16:439-444

2. Gill KR, Camellini L, Conigliaro R, et al. The natural history of upper gastrointestinal subepithelial tumors: a multicenter endoscopic ultrasound survey. J Clin Gastroenterol 2009;43:723-726.

3. Kim MY, Jung HY, Choi KD, et al. Natural history of asymptomatic small gastric subepithelial tumors. J Clin Gastroenterol 2011;45:330-336.

4. Kawanowa K, Sakuma Y, Sakurai S, et al. High incidence of microscopic gastrointestinal stromal tumors in the stomach. Hum Pathol 2006;37:1527-1535.

5. Agaimy A, Wünsch PH, Dirnhofer S, Bihl MP, Terracciano LM, Tornillo L. Microscopic gastrointestinal stromal tumors in esophageal and intestinal surgical resection specimens: a clinicopathologic, immunohistochemical, and molecular study of 19 lesions. Am J Surg Pathol 2008;32:867-873.

6. Miettinen M, Lasota J. Gastrointestinal stromal tumors: pathology and prognosis at different sites. Semin Diagn Pathol 2006;23:70-83.

7. Joensuu H. Risk stratification of patients diagnosed with gastrointestinal stromal tumor. Hum Pathol 2008;39:1411-1419.

8. Miettinen M, Sobin LH, Lasota J. Gastrointestinal stromal tumors of the stomach: a clinicopathologic, immunohistochemical, and molecular genetic study of 1765 cases with long-term follow-up. Am J Surg Pathol 2005;29:52-68.

9. Tryggvason G, Gíslason HG, Magnússon MK, Jónasson JG. Gastrointestinal stromal tumors in Iceland, 1990-2003: the icelandic GIST study, a population-based incidence and pathologic risk stratification study. Int J Cancer 2005;117:289-293.

10. Fletcher CD, Berman JJ, Corless C, et al. Diagnosis of gastrointestinal stromal tumors: a consensus approach. Hum Pathol 2002;33:459-465.

11. Trupiano JK, Stewart RE, Misick C, Appelman HD, Goldblum JR. Gastric stromal tumors: a clinicopathologic study of 77 cases with cor- 
relation of features with nonaggressive and aggressive clinical behaviors. Am J Surg Pathol 2002;26:705-714.

12. Nilsson B, Bümming P, Meis-Kindblom JM, et al. Gastrointestinal stromal tumors: the incidence, prevalence, clinical course, and prognostication in the preimatinib mesylate era: a population-based study in western Sweden. Cancer 2005;103:821-829.

13. Demetri GD, von Mehren M, Antonescu CR, et al. NCCN Task Force report: update on the management of patients with gastrointestinal stromal tumors. J Natl Compr Canc Netw 2010;8(Suppl 2):S1-S41.

14. Lachter J, Bishara N, Rahimi E, Shiller M, Cohen H, Reshef R. EUS clarifies the natural history and ideal management of GISTs. Hepatogastroenterology 2008;55:1653-1656

15. Khashab MA, Pasricha PJ. Conquering the third space: challenges and opportunities for diagnostic and therapeutic endoscopy. Gastrointest Endosc 2013;77:146-148.

16. Meesters B, Pauwels PA, Pijnenburg AM, Vlasveld LT, Repelaer van Driel OJ. Metastasis in a benign duodenal stromal tumour. Eur J Surg Oncol 1998;24:334-335.

17. Nishida T, Kawai N, Yamaguchi S, Nishida Y. Submucosal tumors: comprehensive guide for the diagnosis and therapy of gastrointestinal submucosal tumors. Dig Endosc 2013;25:479-489.
18. ASGE Standards of Practice Committee, Gan SI, Rajan E, et al. Role of EUS. Gastrointest Endosc 2007;66:425-434.

19. Hwang JH, Saunders MD, Rulyak SJ, Shaw S, Nietsch H, Kimmey MB. A prospective study comparing endoscopy and EUS in the evaluation of GI subepithelial masses. Gastrointest Endosc 2005;62:202-208.

20. Karaca C, Turner BG, Cizginer S, Forcione D, Brugge W. Accuracy of EUS in the evaluation of small gastric subepithelial lesions. Gastrointest Endosc 2010;71:722-727.

21. Palazzo L, Landi B, Cellier C, Cuillerier E, Roseau G, Barbier JP. Endosonographic features predictive of benign and malignant gastrointestinal stromal cell tumours. Gut 2000;46:88-92

22. Dumonceau JM, Polkowski M, Larghi A, et al. Indications, results, and clinical impact of endoscopic ultrasound (EUS)-guided sampling in gastroenterology: European Society of Gastrointestinal Endoscopy (ESGE) Clinical Guideline. Endoscopy 2011;43:897-912.

23. ESMO/European Sarcoma Network Working Group. Gastrointestinal stromal tumours: ESMO Clinical Practice Guidelines for diagnosis, treatment and follow-up. Ann Oncol 2014;25 Suppl 3:iii21-iii26.

24. Nishida T, Hirota S, Yanagisawa A, et al. Clinical practice guidelines for gastrointestinal stromal tumor (GIST) in Japan: English version. Int J Clin Oncol 2008;13:416-430. 